\title{
Millimeter-Wave 3D-Printed Antenna Array based on Gap- Waveguide Technology and Split E-plane Waveguide
}

This paper was downloaded from TechRxiv (https://www.techrxiv.org).

\section{LICENSE}

CC BY-NC-SA 4.0

SUBMISSION DATE / POSTED DATE

$25-01-2021 / 26-01-2021$

\section{CITATION}

Palomares-Caballero, Ángel; Alex-Amor, Antonio; Valenzuela-Valdés, Juan; Padilla, Pablo (2021): MillimeterWave 3D-Printed Antenna Array based on Gap-Waveguide Technology and Split E-plane Waveguide. TechRxiv. Preprint. https://doi.org/10.36227/techrxiv.13637924.v1

$\mathrm{DOI}$ 


\section{Copyright:}

(C2021 IEEE. Personal use of this material is permitted. Permission from IEEE must be obtained for all other uses, in any current or future media, including reprinting/republishing this material for advertising or promotional purposes, creating new collective works, for resale or redistribution to servers or lists, or reuse of any copyrighted component of this work in other works.

\section{Disclaimer:}

This work has been published on IEEE Transactions on Antennas and Propagation. DOI: 10.1109/TAP.2020.3008620 


\title{
Millimeter-Wave 3D-Printed Antenna Array based on Gap-Waveguide Technology and Split E-plane Waveguide
}

\author{
Ángel Palomares-Caballero, Antonio Alex-Amor, Juan Valenzuela-Valdés, and Pablo Padilla
}

\begin{abstract}
A multilayer aperture antenna array in millimeterwave band is presented in this article. The antenna array is based on glide-symmetric holey gap-waveguide technology combined with E-plane insertion gaps for a low-cost and low-loss design. The radiating part of the antenna array is formed by an array of sixteen aperture antennas, grouped in four sets of $2 \times 2$ antenna subarrays in E-plane configuration. The $2 \times 2$ subarrays are fed by a one-to-four corporate feeding network in E-plane with holey gap-waveguide technology. The antenna array has been manufactured with high precision stereolithography (SLA) and subsequent metal plating. This design procedure yields a low-cost and low-weight manufacturing process for functional prototypes. The complete array has been manufactured and measured, comparing its performance with the simulation results. Measurements show an input reflection coefficient below -10 dB which ranges from $68 \mathrm{GHz}$ to $74 \mathrm{GHz}$. The measured radiation patterns suit adequately the defined ones in the design stage. Moreover, gain above $19 \mathrm{dBi}$ in the entire operating frequency band is achieved with a $\mathbf{7 4 . 1 \%}$ mean antenna efficiency.
\end{abstract}

Index Terms-Aperture antenna array, gap-waveguide technology, millimeter-wave, stereolithography (SLA).

\section{INTRODUCTION}

Millimeter-wave bands are promising frequency ranges for future communication systems [1]. Antennas at these frequencies provide high directivity to compensate free space propagation losses. However, the manufacturing of millimeterwave antennas is challenging since high accuracy and perfect assembly are needed. Recently, gap-waveguide technology has arisen as a solution for accomplishing the latter conditions with low-loss performance and low manufacturing cost [2]. Several slotted arrays based on gap-waveguide technology have been reported [3]-[9]. In them, the use of pin latticebased metasurface structures is introduced to define the guiding structures of the array [10], [11]. However, the increase of frequency at the millimeter-wave bands forces the pin structures to become smaller and hard to be manufactured.

This work was supported in part by the Spanish Program of Research, Development, and Innovation under Project TIN2016-75097-P,Project RTI2018102002-A-I00, and Project EQC2018-004988-P, in part by the "Junta de Andalucía" under Project B-TIC-402-UGR18 andProject P18.RT.4830, and in part by the Predoctoral Grant FPU18/01965. (Corresponding author: Ángel Palomares-Caballero.)

Á. Palomares-Caballero, A. Alex-Amor, J. Valenzuela-Valdés and P. Padilla are with the Department of Signal Theory, Telematics and Communications, Universidad de Granada, 18071 Granada, Spain (e-mail: angelpc@ugr.es; aalex@ugr.es; juanvalenzuela@ugr.es; pablopadilla@ugr.es ).

A. Alex-Amor is with the Information Processing and Telecommunications Center, Universidad Politécnica de Madrid, 28040 Madrid, Spain (e-mail: aalex@gr.ssr.upm.es).

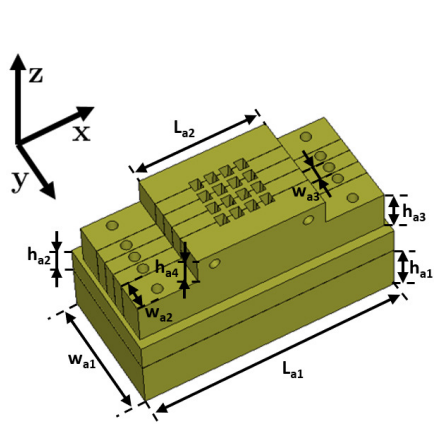

(a)

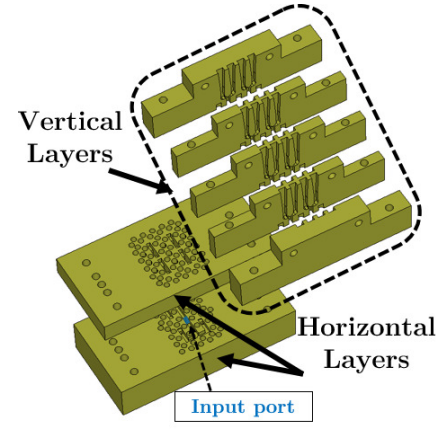

(b)
Fig. 1. Multilayer aperture antenna array in millimeter-wave band: (a) Assembled array view, and (b) The different layers that conform it. Dimensions: $\mathrm{L}_{\mathrm{a} 1}=60 \mathrm{~mm}, \mathrm{~L}_{\mathrm{a} 2}=30 \mathrm{~mm}, \mathrm{w}_{\mathrm{a} 1}=30 \mathrm{~mm}, \mathrm{w}_{\mathrm{a} 2}=6.9 \mathrm{~mm}, \mathrm{w}_{\mathrm{a} 3}=3.49 \mathrm{~mm}$, $\mathrm{h}_{\mathrm{a} 1}=11.25 \mathrm{~mm}, \mathrm{~h}_{\mathrm{a} 2}=6.35 \mathrm{~mm}, \mathrm{~h}_{\mathrm{a} 3}=10 \mathrm{~mm}, \mathrm{~h}_{\mathrm{a} 4}=6.37 \mathrm{~mm}$.

Gap-waveguide technology based on glide-symmetric holes presents an advantage in regard to pin structure manufacturing at such frequencies [12]. A variety of guiding, filtering and phase shifting structures have been designed using this type of gap waveguide technology [13]- [16] with high performance in terms of propagation losses and cost-effective fabrication. However, only [17] presents an antenna design based on this novel gap-waveguide technology.

All the previous works have been manufactured with metallic milling, which leads to a higher cost compared to incoming $3 \mathrm{D}$ printing techniques. Specifically, the 3D printing technique based on stereolithography (SLA) is an adequate candidate for manufacturing high-precision and low-weight prototypes at millimeter-wave bands [18], [19]. Some works make use of this manufacturing technique to design high-frequency arrays [20]- [22]. A subsequent metallic plating stage is needed after SLA manufacturing. Another attractive 3D printing technique is direct metal laser sintering (DMLS). Antenna arrays in [23]- [25] apply this manufacturing technique. The principal disadvantage of DMLS is the surface roughness that is not negligible and produces high losses at upper bands of the millimeter-wave frequency range.

In this document, we present an aperture antenna array based on glide-symmetric gap-waveguide technology centered at $70 \mathrm{GHz}$. The operating frequency band ranges from 66 to 74 GHz. This range covers both access and backhaul millimeterwave applications for 5G [26]. It is included in a band of interest for $5 \mathrm{G}$ above the WiGig (Wireless Gigabit Alliance) frequency band targets to high speed indoor applications. This 
wide $5 \mathrm{G}$ frequency band for millimeter-wave is composed by two bands. The first frequency band, ranging from 66 to $71 \mathrm{GHz}$, is addressed to the radio access network for $5 \mathrm{G}$ connections since the propagation losses at this frequency band is lower than its adjacent band $(57-66 \mathrm{GHz})$. The second band, ranging from $71-76 \mathrm{GHz}$, is intended to support fixed highspeed connection for different applications such as macro or micro cell backhaul links, and front haul connection between a base station and theirs remote radio heads to extend the coverage area.

The design consists on a multilayer design composed by means of stacking horizontal and vertical layers, as shown in Fig. 1. The vertical and horizontal layer gaps are located in the E-plane and the H-plane of the gap-waveguide elements, respectively. Layers with the gap in the H-plane need the gap-waveguide technology to avoid field leakage throughout the gap in millimeter-wave frequencies. Four $2 \times 2$ aperture antenna subarrays conform the radiating part that is fed by a corporate feeding network. A fully-functional prototype has been manufactured in SLA with subsequent copper plating, and conveniently measured for its validation. The relevance and novelty of our work is based on two main aspects. First, we propose a novel design antenna array that uses holey glide-symmetric gap-waveguide and E-plane waveguide structure to reduce the propagation losses in a multilayer array design. In our work, we propose a feasible multilayer design array with a combination between the traditionally used split H-plane waveguide structures with glide symmetric holes, and split E-plane waveguide structures that does not require such elements. Secondly, we demostrate the potential use of SLA to fabricate multilayer array design at millimeter-wave frequencies. Although multilayer array prototoypes based on gap-waveguide technology are commonly manufactured in CNC because of higher accuracy compared to traditional 3-D printing techniques, with the appearance of SLA technique, a high-precision and cost-efective fabrication is possible [19]. The document is organized as follows. Section II depicts the principal elements that constitutes the antenna array design. In this section gap-waveguide technology selected, the corporate feeding network and the subarrays are conveniently described and simulated. Section III provides the measurements of the manufactured prototype and the discussion of the results. Finally, the conclusions are detailed in Section IV.

\section{ANTENNA ARRAY DESIGN}

The antenna array design is formed by two horizontal layers and five vertical layers. All of them are stacked and not welded but assembled with mechanical pressure by screws. Thus, in the array assembling, there are inevitably gaps between layers of at least some tens of microns. Therefore, the gap-waveguide technology and E-plane configurations are adopted to avoid any field leakage due to the layer gaps. Note that gaps are considered between all stacked layers in the design process.

\section{A. Glide-symmetric Holey Gap-waveguide Technology}

Gap-waveguide technology based on glide-symmetric holes provides a wide stopband in a cost-effective way [13]. The

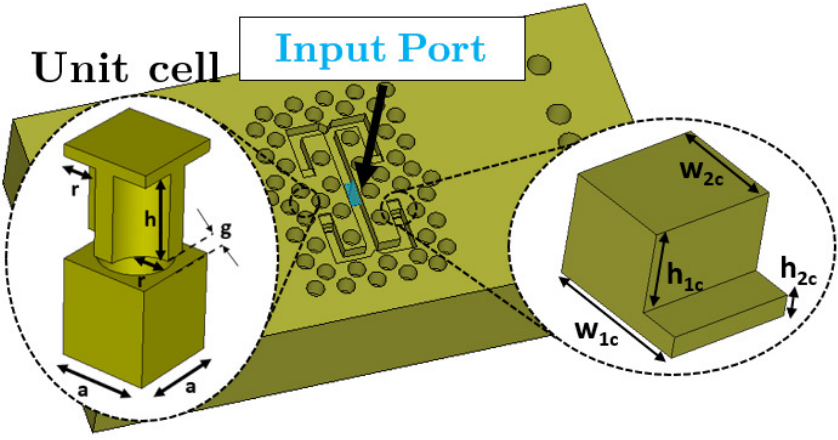

(a)

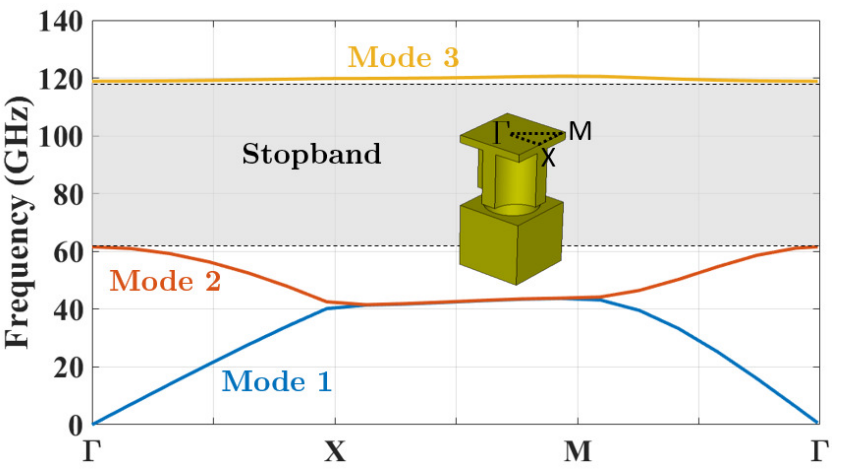

(b)

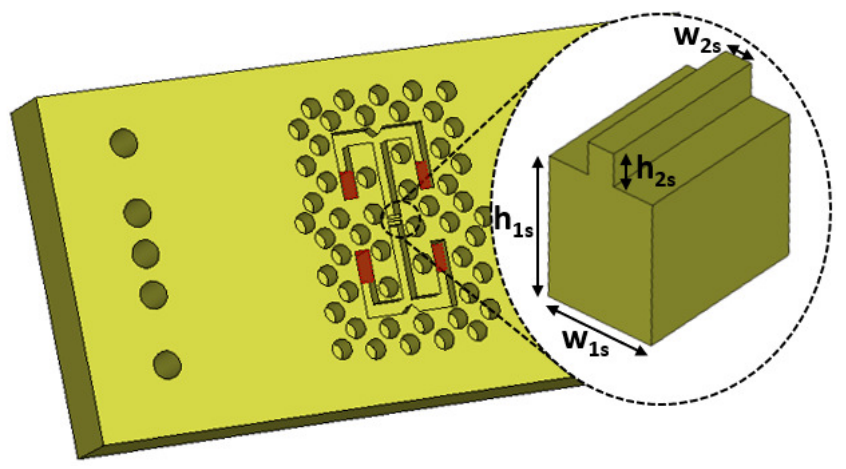

(c)

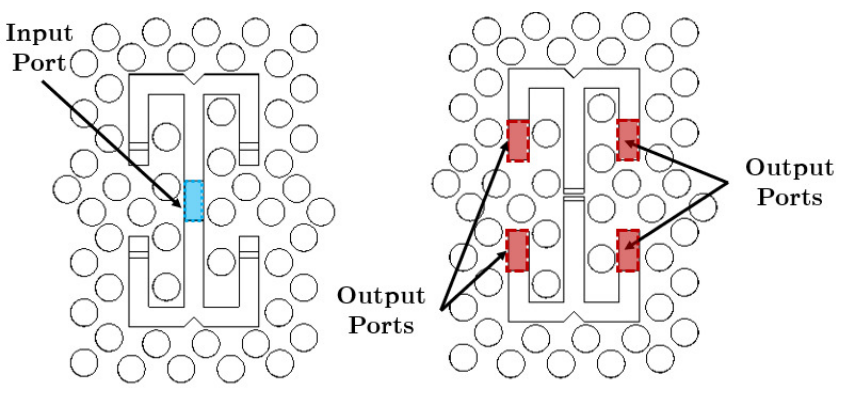

(d)

Fig. 2. Design of the corporate feeding network: (a) Bottom part $\left(1^{\text {st }}\right.$ horizontal layer), $\mathrm{w}_{1 \mathrm{c}}=1.4 \mathrm{~mm}, \mathrm{~h}_{1 \mathrm{c}}=0.75 \mathrm{~mm}, \mathrm{w}_{2 \mathrm{c}}=1 \mathrm{~mm}, \mathrm{~h}_{2 \mathrm{c}}=0.2$ $\mathrm{mm}$; (b) Dispersion diagram of the employed EBG unit cell; (c) Upper part ( $2^{\text {nd }}$ horizontal layer), $\mathrm{w}_{1 \mathrm{~s}}=0.85 \mathrm{~mm}, \mathrm{~h}_{1 \mathrm{~s}}=1 \mathrm{~mm}, \mathrm{w}_{2 \mathrm{~s}}=0.25 \mathrm{~mm}, \mathrm{~h}_{2 \mathrm{~s}}=$ $0.2 \mathrm{~mm}$; and, (d) Planar views of bottom and upper parts.

electromagnetic bandgap (EBG) unit cell is shown in Fig. 2(a). The dimensions used for our simulated EBG structure are: a $=2.26 \mathrm{~mm}, \mathrm{r}=0.88 \mathrm{~mm}, \mathrm{~h}=2.2 \mathrm{~mm}$ and $\mathrm{g}=0.03 \mathrm{~mm}$. The considered gap size is in the range of reported multi- 
layer antennas at millimeter-wave frequencies. The provided stopband of the EBG unit cell ranges from $62 \mathrm{GHz}$ to 118 $\mathrm{GHz}$ as it is shown in Fig. 2(b). The unit cell dimensions are chosen in such a way that they fit inside the corporate feeding network and at the same time the stopband is included in the target operating frequency band $(66-74 \mathrm{GHz})$. This is why the lowest frequency limit of the stopband is close to the start operating frequency.

\section{B. 1-to-4 Corporate Feeding Network}

Figs. 2(a), 2(c) and 2(d) show the two halves that form the corporate feeding network both in 3D and planar views. The waveguides that compose this design and the feeding port (input port) have WR-10 waveguide dimensions. The input port is located at the bottom of the first horizontal layer, as shown in Fig. 2(a) and Fig. 2(d). Each horizontal layer of the feeding network occupies half of the width of the waveguides. When the layers are stacked, they form a feeding network with split E-plane waveguides. The gap between layers is preserved in the middle of the wider side of the waveguide (E-plane) except in the H-plane divider located next the input port and in the $90^{\circ}$ bends before the output ports. Because there are locations in the feeding network where the gap is not kept in the E-plane, glide-symmetric holey gap-waveguide is implemented between layers. The feeding network distributes in-phase and equally the input power among the output ports (highlighted in red). These ports connect the feeding network with the four subarrays. The matching elements in the power dividers and the $90^{\circ}$ bends are shown in detail in Figs. 2(a), 2(c) and 2(d).

The simulated performance of the feeding network design is illustrated in Fig. 3. The reflection coefficient is maintained

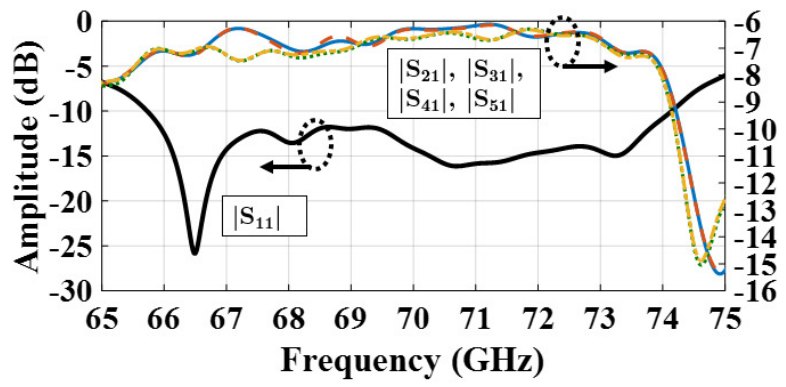

(a)

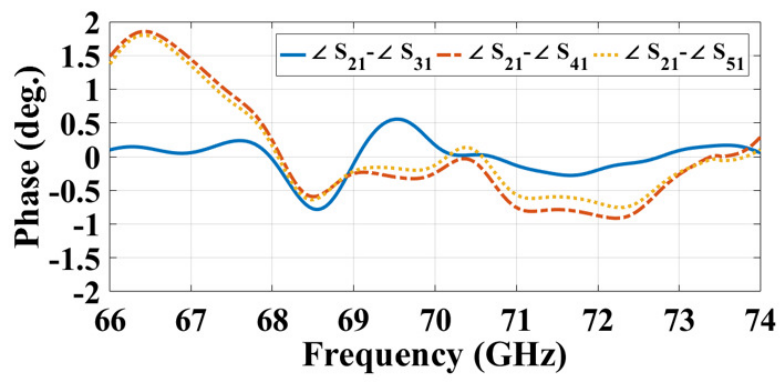

(b)

Fig. 3. Performance of the corporate feeding network: (a) Amplitude of the S-parameter simulated results, (b) Phase imbalance between the output ports. below $-10 \mathrm{~dB}$ from $66 \mathrm{GHz}$ to $74 \mathrm{GHz}$, with an approximately balanced and in-phase transmission coefficients. The maximum amplitude and phase variations are $1 \mathrm{~dB}$ and $1.8^{\circ}$, respectively. Although the feeding network has E-plane power dividers, the in-phase output fields are obtained due to the presence of E-plane bends before the output ports.

\section{C. $2 x 2$ Aperture Antenna Subarray}

The configuration of the $2 \times 2$ aperture antenna subarrays is shown in Figs. 4(a) and 4(b). The power division from the output ports of the corporate feeding network to the aperture antennas is carried out by H-plane and E-plane power dividers as they are illustrated in Fig. 4(c). The H-plane power divider is located at the joint between the second horizontal layer and the vertical layers and just above the output ports of the feeding network. This fact is observed on the left part of Fig. 4(c). E-plane power dividers are implemented before the antenna apertures as it is illustrated on the right part of Fig. 4(c). These power dividers design are formed by stacking the vertical layers in the horizontal direction as it is shown in Figs. 4(a) and 4(b). This stack of layers does not need any gap-waveguide technology since the gap is preserved in the E-plane location of the waveguide belonging to subarray antenna design. Absence of leakage is only produced by split E-plane waveguides because it does not exist disruption of the surface currents. The array antennas are E-plane aperture antennas with a separation between them of $0.87 \lambda_{0}$ in $x$ and $y$ directions, where $\lambda_{0}$ is the free-space wavelength at $74 \mathrm{GHz}$.

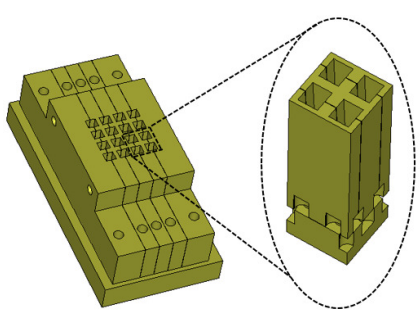

(a)

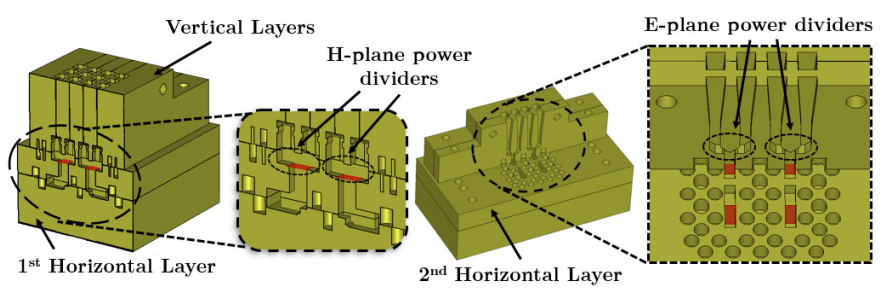

(c)

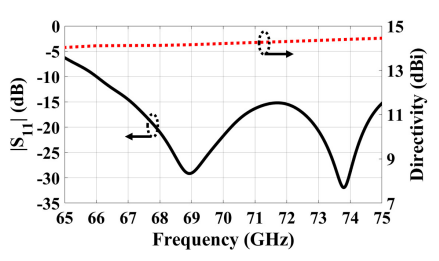

(d)

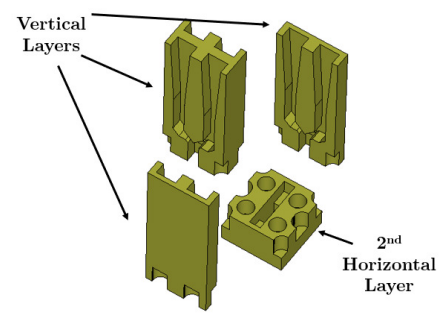

(b)

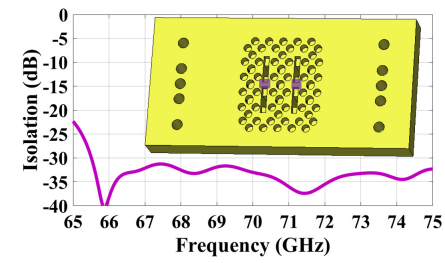

(e)
Fig. 4. Subarray: (a) Assembled view, (b) Exploded view, (c) E-plane and H-plane power dividers locations, (d) Simulated reflection coefficient and directivity, and (e) Simulated isolation level between subarrays. 
The simulation results in periodic conditions for the subarray design are illustrated in Fig. 4(d). An impedance matching under $-10 \mathrm{~dB}$ is obtained in the complete operating frequency range with a stable directivity in frequency of $14 \mathrm{dBi}$.

Glide-symmetric EBG holes are also necessary between the upper part of the second horizontal layer and the bottom part of each vertical layer. This need is due to the existing gap that surrounds the H-plane power dividers. However, there are areas where holes cannot be placed, as Fig. 4(e) shows highlighted. Despite that, more than $30 \mathrm{~dB}$ of isolation level between subarrays is achieved in the entire frequency range. Fig. 5 illustrates an E-field comparison between the antenna array design with and without the implementation of the gap-waveguide technology. The electric field at different frequencies inside the gap between the first and the second horizontal layers for both designs, with gap-waveguide and without gap-waveguide technology, are shown in Figs. 5(c) and 5(d), respectively. Gap size corresponding to the EBG unit cell has been introduced between layers in both antenna designs for a fair comparison. It is clearly seen the importance of the glide-symmetric holes to not produce leakage and thus, loss in antenna efficiency as illustrated in Fig. 5(e).

\section{Details of the gap-waveguide performance and field con- finement}

For the sake of completeness, two cases of study have been carried out for the selected EBG unit cell and waveguide dimensions. They demonstrate the beneficial use of this gapwaveguide technology when a split waveguide is designed. It is important to note that the structures with and without gapwaveguide have the same gap of $30 \mu \mathrm{m}$. The first comparison is shown in Fig. 6 between a E-plane split waveguide with the use of gap-waveguide technology (marked in red) and without gap-waveguide technology (marked in blue). In this case the gap is in the ZY plane and it is displaced from the center of the wide side of the waveguide since if the gap is located at the center, no leakage occurs. The S-parameters are illustrated in Fig. 6(b). A drop of the transmission coefficient is observed in the design without gap-waveguide. In Fig. 6(c) the electric fields reveal the absence of leakage when holey EBG structures are employed. Conversely, field resonances appear in the gap region due to the leakage provoked by the absence of EBG holes (case without gap waveguide). Note that the electric field in this figure is represented in absolute value in order to observe the electric field in the waveguide (oriented along $z$ direction) and in the gap (oriented along $x$ direction since it is a parallel-plate mode). The second comparison corresponds to the H-plane bend implemented in the proposed array before the outputs of the feeding network (Fig. 4(c)). The gap is in the ZY plane as it is observed in Fig. 7(a). The transmission coefficient for the case in which the gap-waveguide technology is not used (Fig. 7(b)) suffers a drop in the transmission coefficient which does not occur for the case with gap-waveguide technology. In addition, Fig. 7(c) confirms these losses and the existence of leakage when the gap-waveguide technology is not employed. Since the proposed array has zones where the gap is not located in the center of the wide side of the waveguide,

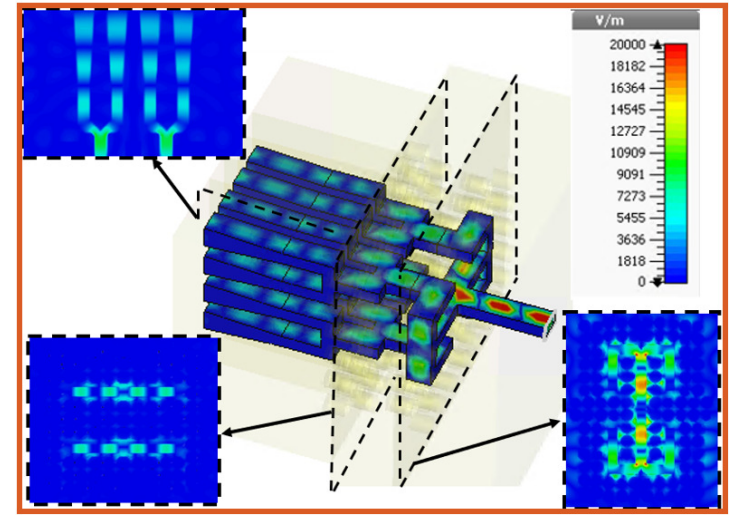

(a)

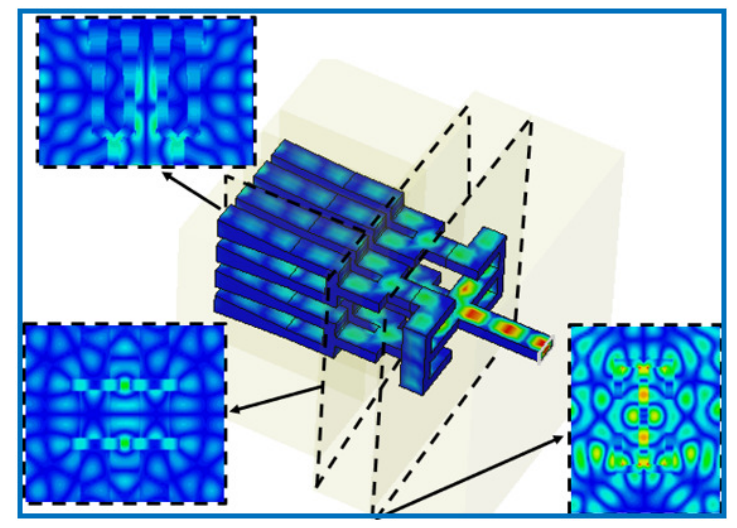

(b)

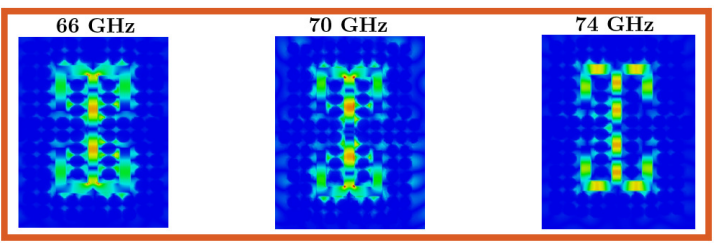

(c)

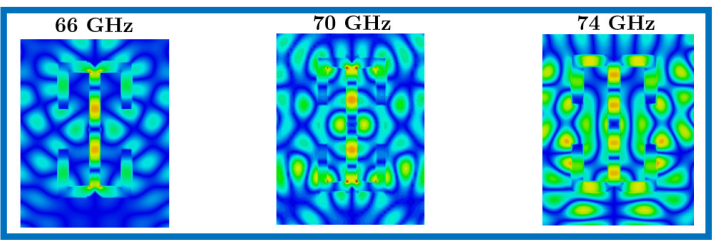

(d)

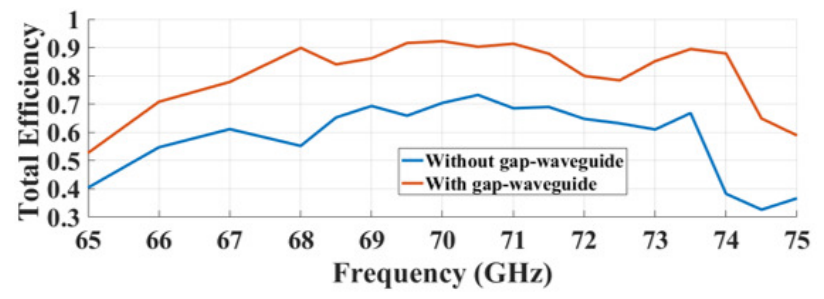

(e)

Fig. 5. Simulated E-field distribution inside the antenna array and between layers at $70 \mathrm{GHz}$ : (a) With gap-waveguide and, (b) Without gap-waveguide. Electric field at different frequencies in the gap between horizontal layers of the antenna array for: (c) The design with gap-waveguide technology and, (d) The design without gap-waveguide technology. (e) Total antenna efficiency comparison. 

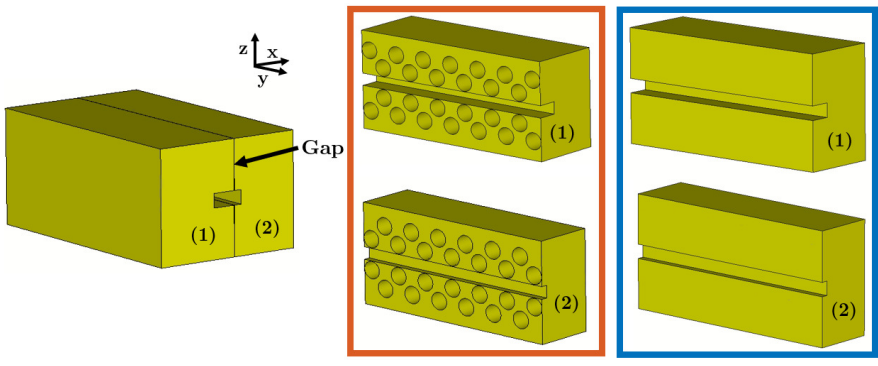

(a)

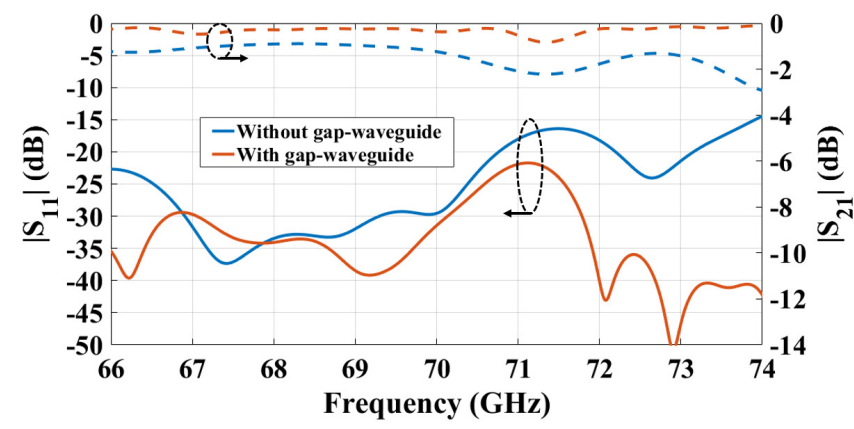

(b)

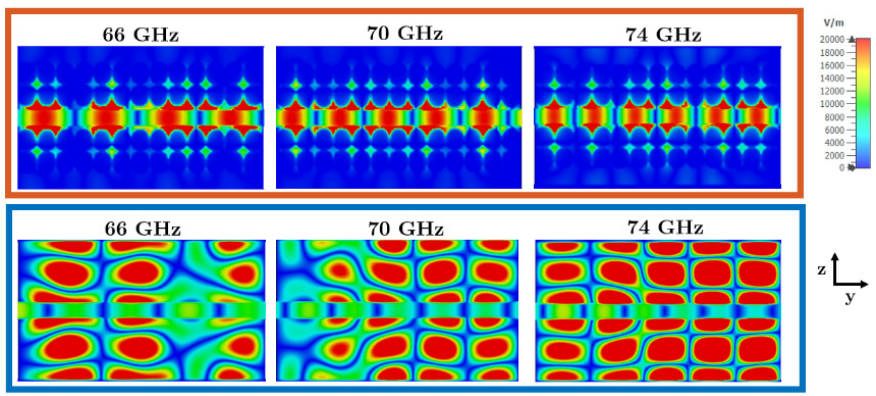

(c)

Fig. 6. Performance comparison between a E-plane split waveguide with gap-waveguide and without gap-waveguide: (a) Design and forming layers, (b) Simulated S-parameters and, (c) Electric field at different frequencies in the gap.

the application of gap-waveguide technology is mandatory to avoid leakage throughout the gap.

\section{MANUfacturing, MEASUREMENTS AND DISCUSSION}

High-precision SLA with a posterior copper plating stage was the selected manufacturing technique due to its low cost compared to milling fabrication. The used plastic material and type of plating for the fabrication are Accura Xtreme White [27] and electroplating, respectively. Fig. 8 shows different views of the fabricated antenna array. The antenna is composed by 7 different forming layers to guarantee a proper metal plating. Detail view on the manufactured parts of the antenna array is shown in Fig. 8(c). Good geometry definitions are observed under the microscope. An extension of the size of the antenna array was needed to place the holes of the assembling screws which in spite of their size do not affect the radiation pattern measurements. Moreover, the first horizontal layer was thicken in order to allow the screwed connection with the waveguide flange. The dimensions of the antenna array design without the assembling parts are $15.8 \times 23.35 \times 22.8$

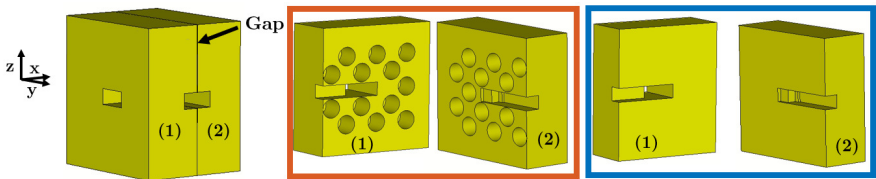

(a)

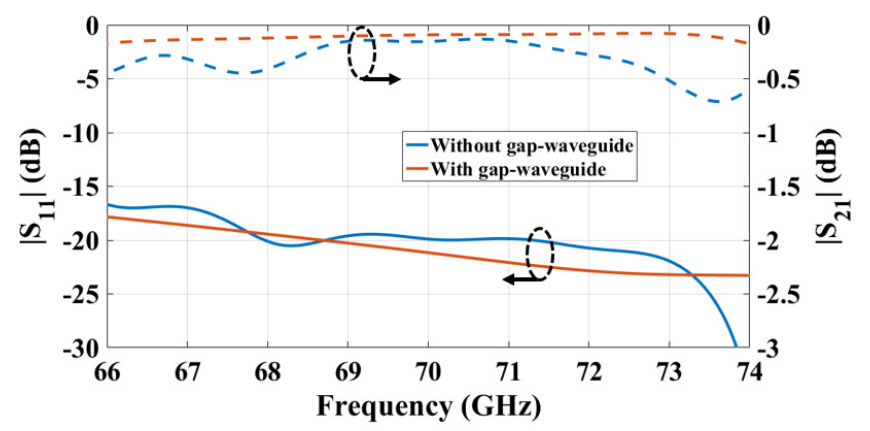

(b)

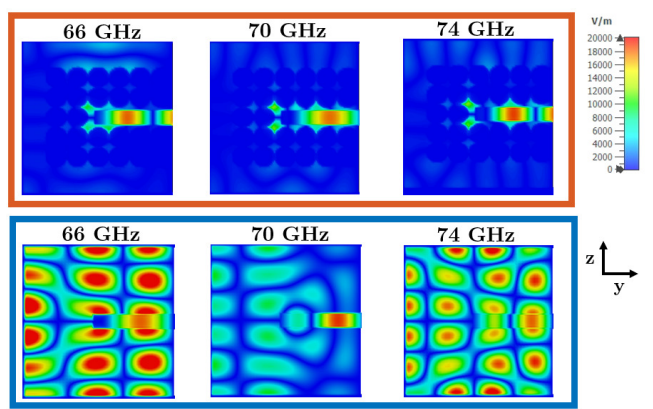

(c)

Fig. 7. Performance comparison between a H-plane bend waveguide with gap-waveguide and without gap-waveguide: (a) Design and forming layers, (b) Simulated S-parameters and, (c) Electric field at different frequencies in the gap.

$\mathrm{mm}$. The reflection coefficient was measured with R\&S ZVAZ110E Converters from $65 \mathrm{GHz}$ to $75 \mathrm{GHz}$. Fine sampling has been applied to reveal any possible glitch caused by poor contact between forming layers. The measurement is compared to the simulation results in Fig. 9. The measured reflection coefficient presents a shift in the lowest frequency of the operating band, from $66 \mathrm{GHz}$ to $68 \mathrm{GHz}$, preserving the rest of the operating band below $-10 \mathrm{~dB}$. To determine the cause of the $\left|S_{11}\right|$ modification with respect to the simulated one, a variation analysis based on the manufacturing tolerances is carried out. An assembly tolerance that taking into account the misalignment between forming layers of the feeding network is also introduced in the variation analysis. This tolerance has been selected since it is the antenna array part where there are more zones with the gap not placed in the Eplane of the waveguide. The variation analysis shows that the measurement result is included in the variability range. The antenna simulation with tolerances which best fits the measurement result is highlighted in Fig. 9. The tolerances for this case are: $\operatorname{tol}_{\mathrm{XY}}=+30 \mu \mathrm{m}, \mathrm{tol}_{\mathrm{Z}}=+55 \mu \mathrm{m}$ and $\Delta_{x y}=$ $50 \mu \mathrm{m}$, which correspond to tolerance in XY plane, tolerance in $z$ direction and misalignment between layers, respectively.

Once the $\left|S_{11}\right|$ of the array is measured and discussed, the radiation patterns of the antenna array are measured, 


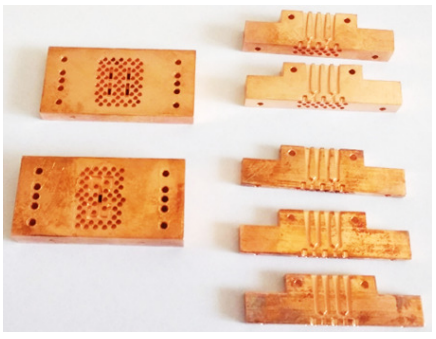

(a)
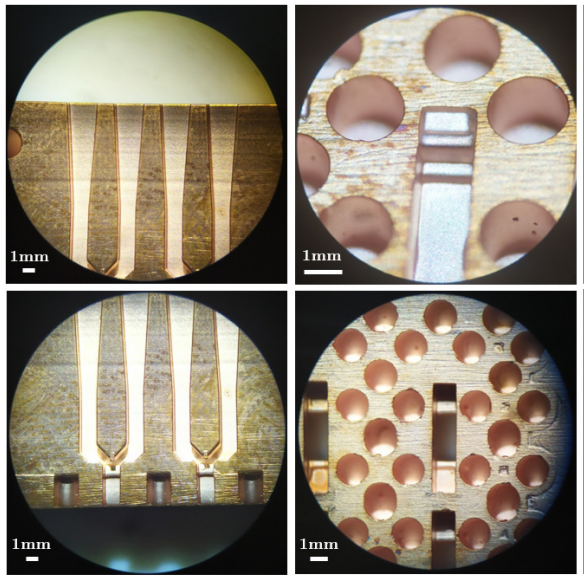

(c)

Fig. 8. Manufactured prototype: (a) Forming layers, (b) Stacked antenna array, and (c) Details under microscope.

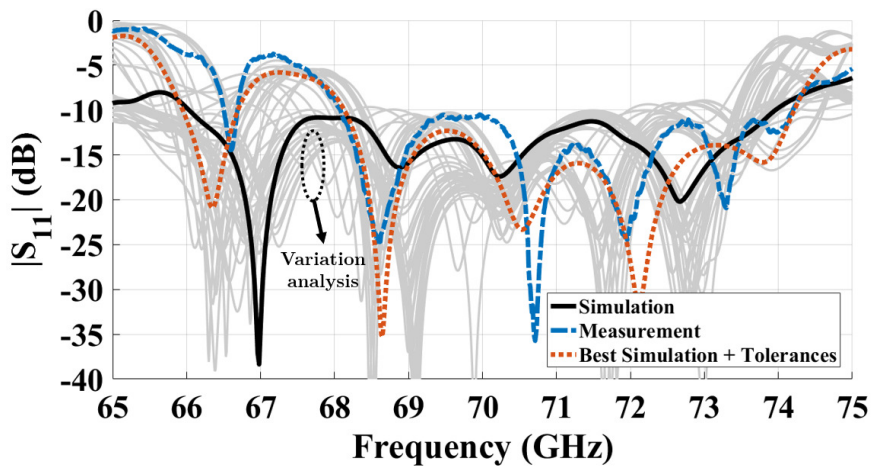

Fig. 9. Comparison of the simulated, simulated with manufacturing tolerances and measured reflection coefficient of the fabricated antenna array. Black line represents the nominal simulation and grey lines show the effect of manufacturing tolerances over the nominal simulation.

for the lowest, center and highest frequency values of the operating band. Those measurements have been carried out at the millimeter-wave antenna measuring facilities of the University of Granada. Fig. 10 depicts the radiation measurement setup. Laser alignment has been used in order to improve the accuracy of the alignment between the probe antenna and the antenna array. The E-plane and H-plane copolar and crosspolar measurements are provided in Fig. 11. The measured radiation patterns and their corresponding simulated values are in good agreement, keeping the beamwidth of the principal lobe and levels for the side lobes below $-12 \mathrm{~dB}$ for all radiation cuts. Crosspolar levels remain under $-22 \mathrm{~dB}$. Fig.

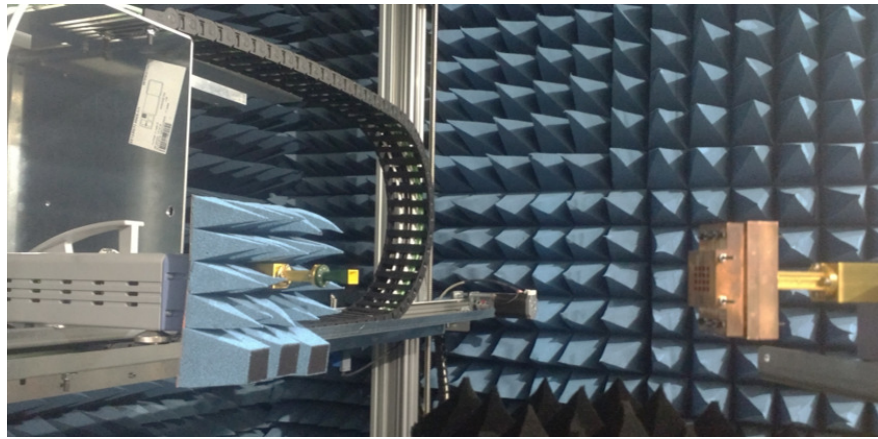

Fig. 10. Detail of the measurement setup at the millimeter-wave antenna measuring facilities of the University of Granada.

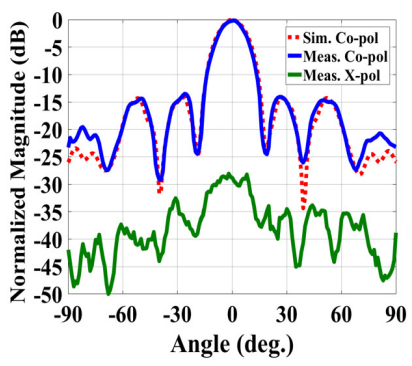

(a)

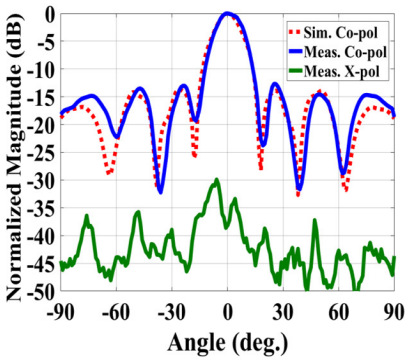

(c)

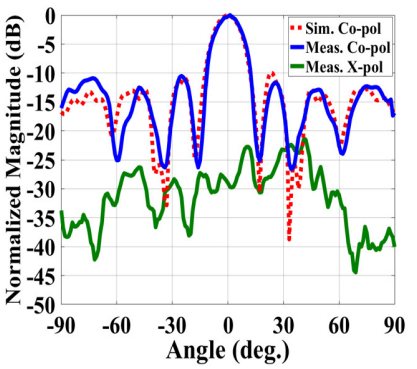

(e)

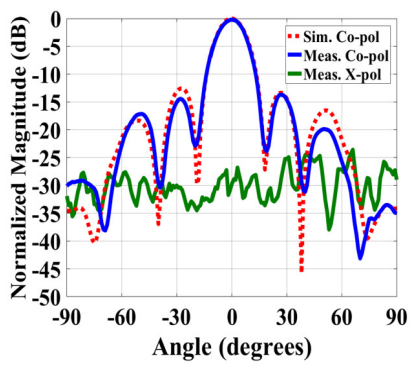

(b)

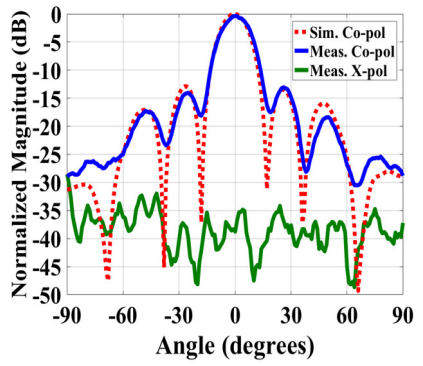

(d)

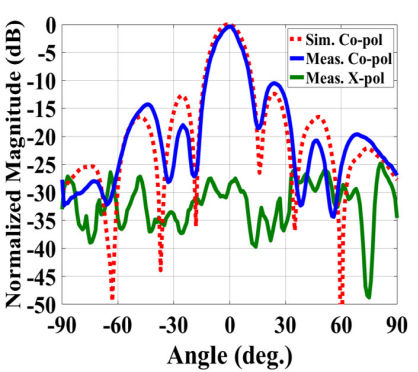

(f)
Fig. 11. Simulated and measured E-plane radiation patterns of the proposed antenna array at different frequencies: (a) $68 \mathrm{GHz}$, (c) $71 \mathrm{GHz}$, and (e) 74 $\mathrm{GHz}$. Simulated and measured H-plane radiation patterns of the proposed antenna array at different frequencies: (b) $68 \mathrm{GHz}$, (d) $71 \mathrm{GHz}$, and (f) 74 $\mathrm{GHz}$. The simulations include the fastening screws.

12 shows the directivity, gain and antenna efficiency values of the antenna array for its entire operating frequency range. For a fair comparison, simulation results correspond to the antenna array with tolerances that best fits the measurements. The measured gain is approximately $20 \mathrm{dBi}$ in the entire frequency range, except above $73 \mathrm{GHz}$, where it drops. Also, the antenna efficiency mean value is $74.1 \%$ that confirms the 


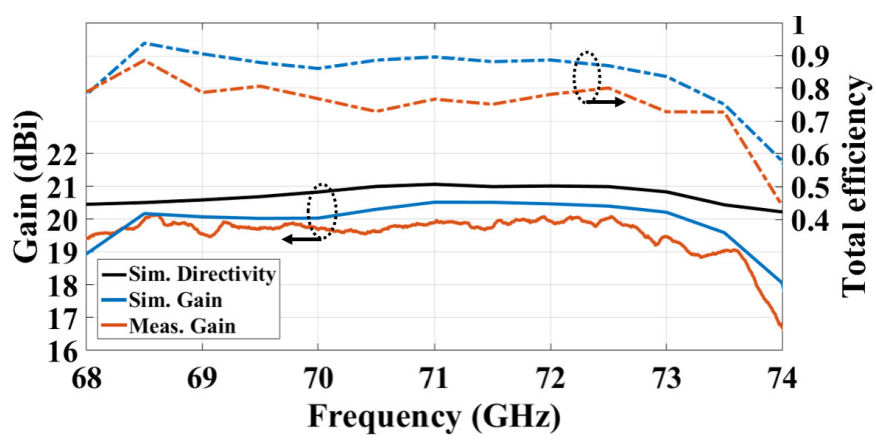

Fig. 12. Radiation performance of proposed antenna array over the operating frequency bandwidth.

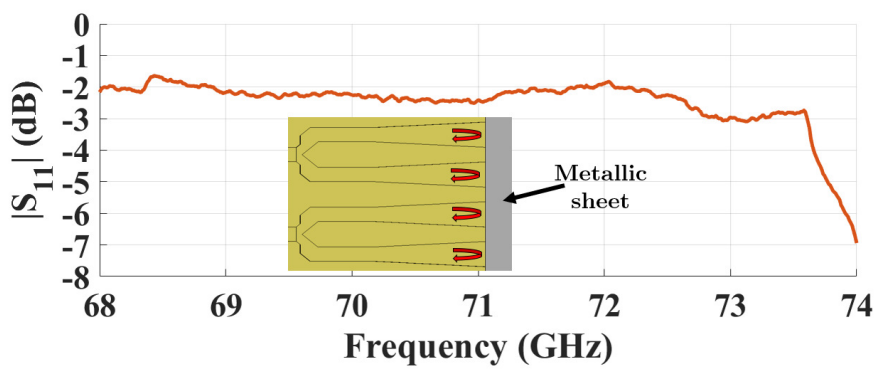

Fig. 13. Measured reflection coefficient when the aperture is short-circuited. This value is twice (round trip) the sum of the ohmic and leakage losses.

correct consideration of the gap value for the EBG unit cell.

In order to verify the existence of leakage in the upper frequency range of the antenna array two kind of measurements are carried out. First one consists of measuring the reflection coefficient of the antenna array when the antennas are short-circuited [28]. This measurement contains twice the insertion losses provided by the fabricated antenna array. Fig. 13 provides the measurement result when the antenna array is short-circuited by a metallic sheet. It is observed that in the high frequency zone part of the operating band, the reflection coefficient value decreases. The estimated losses of the frequency zone where the gain drops are caused by leakage, since the antenna losses in the rest of the band are mainly due to ohmic losses. A maximum of $1 \mathrm{~dB}$ of simulated ohmic losses is obtained for non-ideal copper conductivities. The second measurement to check the presence of leakage is based on 2D far-field radiation patterns. The measurements are illustrated in Fig. 14. They reveal the existence of unwanted radiation from $73 \mathrm{GHz}$ which coincides with the drop in the measured gains. This decrease in gain may be provoked by the imperfect alignment between horizontal layers $\left(\Delta_{x y}\right)$ in the assembly procedure. This parameter is of great importance in the performance of the glide-symmetric holey structures because it affects the stopband of gap waveguide [15]. Since there is a misalignment for the simulation which best fits the measurement, the observed leakage can be explained.

The proposed antenna array is compared with other millimeter-wave antenna arrays in Table I. Same level of antenna gain and efficiency is achieved in this work compared to the state-of-the-art works. The main advantages of our antenna array design are its lower cost, weight and complexity
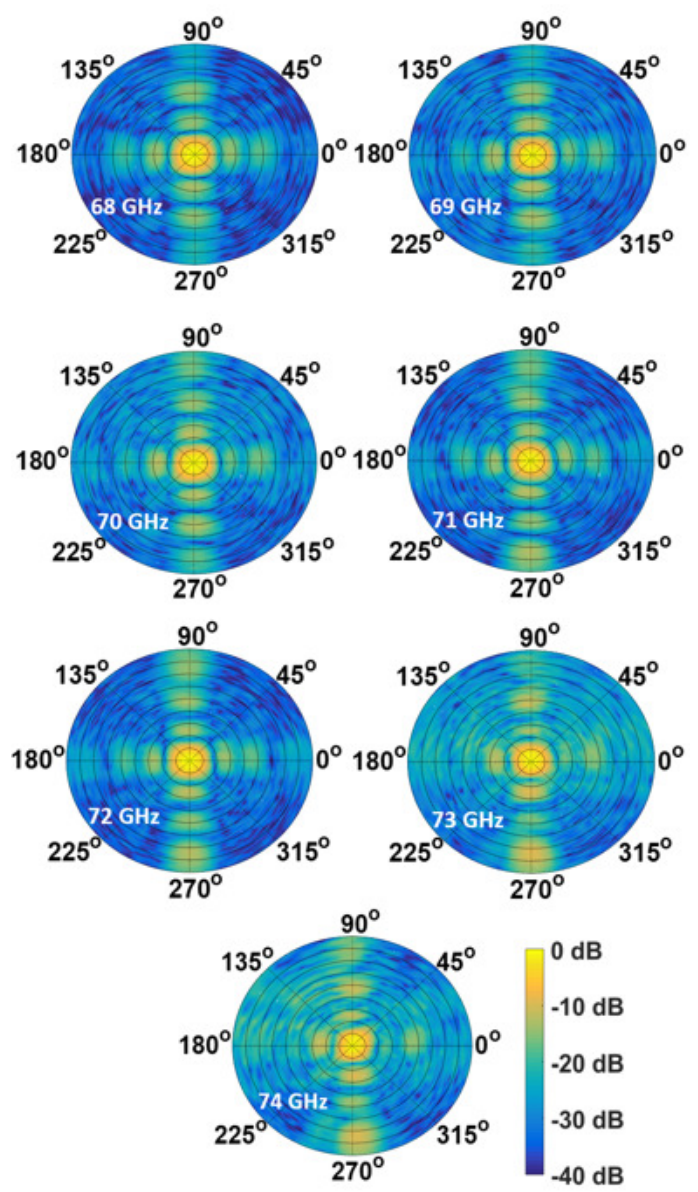

Fig. 14. 2-D far-field radiation pattern of the proposed antenna array at different frequencies.

provided by SLA manufacturing with the combined used of glide-symmetric holey gap-waveguide and split E-plane waveguide structures in a multilayer array antenna. In comparison, CNC milling designs suffer from higher manufacturing complexity than the 3D-printing ones leading to increase the prototype cost. Therefore, this prototype provides an insight of a low-cost and robust solution for multilayer millimeter-wave antenna array designs with gap-waveguide technology.

\section{CONCLUSIONS}

In this work, we have proposed an aperture antenna array design based on gap-waveguide technology and manufactured with high-precision stereolithography process with latter metallic plating. The novel gap-waveguide technology based on glide-symmetric holey structures in combination with split E-plane waveguide enables a multilayer antenna array design for 3-D printed manufacturing. A prototype of the complete $4 \times 4$ aperture antenna array has been manufactured and measured. To best of the authors' knowledge, the antenna array proposed in this paper is the first multilayer gap-waveguide array manufactured by SLA and metal plating with good radiation performance at $70 \mathrm{GHz}$. 
TABLE I

COMPARISON BETWEEN PROPOSED AND REFERRED MILLIMETER-WAVE ANTENNA ARRAYS

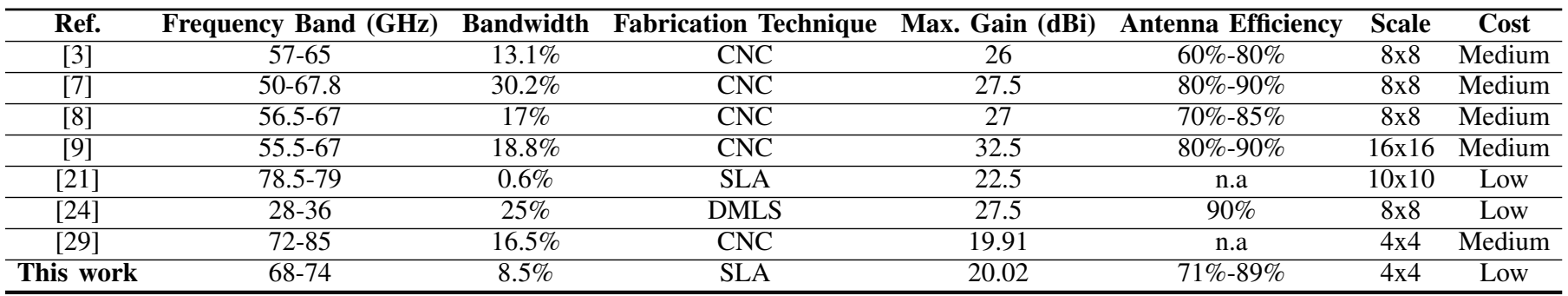

\section{REFERENCES}

[1] Y. Li, E. Pateromichelakis, N. Vucic, J. Luo, W. Xu and G. Caire, "Radio Resource Management Considerations for 5G Millimeter Wave Backhaul and Access Networks," IEEE Commun. Mag., vol. 55, no. 6, pp. 86-92, June 2017.

[2] E. Rajo-Iglesias, M. Ferrando-Rocher and A. U. Zaman, "Gap Waveguide Technology for Millimeter-Wave Antenna Systems," IEEE Commun. Mag., vol. 56, no. 7, pp. 14-20, July 2018.

[3] A. Vosoogh and P. Kildal, "Corporate-Fed Planar 60-GHz Slot Array Made of Three Unconnected Metal Layers Using AMC Pin Surface for the Gap Waveguide," IEEE Antennas Wirel. Propag. Lett., vol. 15, pp. 1935-1938, 2016.

[4] D. Zarifi, A. Farahbakhsh, A. U. Zaman and P. Kildal, "Design and Fabrication of a High-Gain 60-GHz Corrugated Slot Antenna Array With Ridge Gap Waveguide Distribution Layer," IEEE Trans. Antennas Propag., vol. 64, no. 7, pp. 2905-2913, July 2016.

[5] A. Vosoogh, P. Kildal and V. Vassilev, "Wideband and High-Gain Corporate-Fed Gap Waveguide Slot Array Antenna With ETSI Class II Radiation Pattern in V-Band," IEEE Trans. Antennas Propag., vol. 65, no. 4, pp. 1823-1831, April 2017.

[6] A. Jiménez Sáez, A. Valero-Nogueira, J. I. Herranz and B. Bernardo, "Single-Layer Cavity-Backed Slot Array Fed by Groove Gap Waveguide," IEEE Antennas Wirel. Propag. Lett., vol. 15, pp. 1402-1405, 2016.

[7] A. Farahbakhsh, D. Zarifi and A. U. Zaman, "A mmWave Wideband Slot Array Antenna Based on Ridge Gap Waveguide With 30\% Bandwidth," IEEE Trans. Antennas Propag., vol. 66, no. 2, pp. 1008-1013, Feb. 2018.

[8] J. Liu, A. Vosoogh, A. U. Zaman and J. Yang, "A Slot Array Antenna With Single-Layered Corporate-Feed Based on Ridge Gap Waveguide in the $60 \mathrm{GHz}$ Band," IEEE Trans. Antennas Propag., vol. 67, no. 3, pp. 1650-1658, March 2019.

[9] A. Farahbakhsh, D. Zarifi and A. U. Zaman, "60-GHz Groove Gap Waveguide Based Wideband H-Plane Power Dividers and Transitions: For Use in High-Gain Slot Array Antenna," IEEE Trans. Microw. Theory Tech., vol. 65, no. 11, pp. 4111-4121, Nov. 2017.

[10] P. Kildal, E. Alfonso, A. Valero-Nogueira and E. Rajo-Iglesias, "Local Metamaterial-Based Waveguides in Gaps Between Parallel Metal Plates," IEEE Antennas Wirel. Propag. Lett., vol. 8, pp. 84-87, 2009.

[11] A. Berenguer, V. Fusco, D. E. Zelenchuk, D. Sánchez-Escuderos, M. Baquero-Escudero and V. E. Boria-Esbert, "Propagation Characteristics of Groove Gap Waveguide Below and Above Cutoff," IEEE Trans. Microw. Theory Tech., vol. 64, no. 1, pp. 27-36, Jan. 2016.

[12] M. Ebrahimpouri, O. Quevedo-Teruel and E. Rajo-Iglesias, "Design Guidelines for Gap Waveguide Technology Based on Glide-Symmetric Holey Structures," IEEE Microw. Wirel. Compon. Lett., vol. 27, no. 6, pp. 542-544, June 2017.

[13] M. Ebrahimpouri, E. Rajo-Iglesias, Z. Sipus and O. Quevedo-Teruel, "Cost-Effective Gap Waveguide Technology Based on Glide-Symmetric Holey EBG Structures," IEEE Trans. Microw. Theory Tech., vol. 66, no. 2, pp. 927-934, Feb. 2018.

[14] Á. Palomares-Caballero, A. Alex-Amor, P. Padilla, F. Luna and J. Valenzuela-Valdés, "Compact and Low-Loss V-Band Waveguide Phase Shifter Based on Glide-Symmetric Pin Configuration," in IEEE Access, vol. 7, pp. 31297-31304, 2019.

[15] P. Padilla, Á. Palomares-Caballero, A. Alex-Amor, J. Valenzuela-Valdés, J. M. Fernandez-González and O. Quevedo-Teruel, "Broken GlideSymmetric Holey Structures for Bandgap Selection in Gap-Waveguide Technology," IEEE Microw. Wirel. Compon. Lett., vol. 29, no. 5, pp. 327-329, May 2019.
[16] E. Rajo-Iglesias, M. Ebrahimpouri and O. Quevedo-Teruel, "Wideband Phase Shifter in Groove Gap Waveguide Technology Implemented With Glide-Symmetric Holey EBG," IEEE Microw. Wirel. Compon. Lett., vol. 28, no. 6, pp. 476-478, June 2018.

[17] Q. Liao, E. Rajo-Iglesias and O. Quevedo-Teruel, " $K a$-Band Fully Metallic TE40 Slot Array Antenna With Glide-Symmetric Gap Waveguide Technology," IEEE Trans. Antennas Propag., vol. 67, no. 10, pp. 6410-6418, Oct. 2019.

[18] A. Tamayo-Domínguez, J. Fernández-González and M. Sierra-Pérez, "Groove Gap Waveguide in 3-D Printed Technology for Low Loss, Weight, and Cost Distribution Networks," IEEE Trans. Microw. Theory Tech., vol. 65, no. 11, pp. 4138-4147, Nov. 2017.

[19] A. I. Dimitriadis et al., "Polymer-Based Additive Manufacturing of High-Performance Waveguide and Antenna Components," Proc. IEEE, vol. 105, no. 4, pp. 668-676, April 2017.

[20] G. P. Le Sage, "3D Printed Waveguide Slot Array Antennas," IEEE Access, vol. 4, pp. 1258-1265, 2016.

[21] J. Tak, A. Kantemur, Y. Sharma and H. Xin, "A 3-D-Printed W-Band Slotted Waveguide Array Antenna Optimized Using Machine Learning," IEEE Antennas Wirel. Propag. Lett., vol. 17, no. 11, pp. 2008-2012, Nov. 2018.

[22] M. García-Vigueras, E. Menargues, T. Debogovic, E. de Rijk and J. R. Mosig, "Cost-effective dual-polarised leaky-wave antennas enabled by three-dimensional printing," IET Microw. Antennas Propag., vol. 11, no. 14, pp. 1985-1991, 19112017.

[23] E. García-Marín, J. L. Masa-Campos, P. Sánchez-Olivares and J. A. Ruiz-Cruz, "Evaluation of Additive Manufacturing Techniques Applied to Ku-Band Multilayer Corporate Waveguide Antennas," IEEE Antennas Wirel. Propag. Lett., vol. 17, no. 11, pp. 2114-2118, Nov. 2018.

[24] Y. Li et al., "3-D Printed High-Gain Wideband Waveguide Fed Horn Antenna Arrays for Millimeter-Wave Applications," IEEE Trans. Antennas Propag., vol. 67, no. 5, pp. 2868-2877, May 2019.

[25] G. Huang, S. Zhou, T. Chio and T. Yeo, "Fabrication of a HighEfficiency Waveguide Antenna Array via Direct Metal Laser Sintering," IEEE Antennas Wirel. Propag. Lett., vol. 15, pp. 622-625, 2016.

[26] G. Varrall, 5G Spectrum and Standards. Artech House, 2nd Edition, 2016.

[27] 3DSYSTEMS, “Accura Xtreme White 200", 2015. Available: https://www.3dsystems.com/sites/default/files/accura-xtreme-white200-usen.pdf

[28] A. Gomez-Torrent, U. Shah and J. Oberhammer, "Wideband $220-$ $330 \mathrm{GHz}$ Turnstile OMT Enabled by Silicon Micromachining," in 2018 IEEE/MTT-S International Microwave Symposium - IMS, Philadelphia, PA, 2018, pp. 1511-1514

[29] B. Gao, Y. Ren, H. Wu, X. Liao, K. Li and F. Wang, "A Novel CorporateFeed Horn Sub-Array Antenna for the $77 \mathrm{GHz}-B$ and," IEEE Access, vol. 6, pp. 37166-37172, 2018. 